\begin{tabular}{|c|c|}
\hline Title & Onset mechanism of primary acoustic instability in downward-propagating flames \\
\hline Author(s) & Y oon, Sung Hwan; Noh, Tae Joon; Fujita, O samu \\
\hline Citation & $\begin{array}{l}\text { Combustion and flame, } 170,1-11 \\
\text { https://doi.org/10.1016//.combustflame.2016.05.008 }\end{array}$ \\
\hline Issue Date & $2016-08$ \\
\hline Doc URL & http:/hdl.handle.net/2115/71130 \\
\hline Rights & $\begin{array}{l}\text { (2) 2016. This manuscript version is made available under the CC-BY-NC-ND } 4.0 \text { license } \\
\text { http://creativecommons.org/icenses/by-nc-nd/4.0/ }\end{array}$ \\
\hline Rights(URL) & http://creativecommons.org/icenses/by-nc-nd/4.0/ \\
\hline Type & article (author version) \\
\hline File Information & CNF_Y oon\&Fujita_FINAL_VERSION.pdf \\
\hline
\end{tabular}

Instructions for use 


\title{
Onset Mechanism of Primary Acoustic Instability in Downward-Propagating Flames
}

\author{
SungHwan Yoon, TaeJoon Noh, Osamu Fujita* \\ Division of Mechanical and Space Engineering, \\ Graduate School of Engineering, \\ Hokkaido University
}

*Corresponding author: Tel: (+81) 11 7066385; Fax: (+81) 11 7067841;

Email address: ofujita@eng.hokudai.ac.jp;

Postal address: Hokkaido University, Kita13 Nishi 8, Kita-ku, Sapporo, Hokkaido, Japan 


\section{Nomenclature}

$a$

$a_{\mathrm{f}}$

$A_{\mathrm{f}}$

C

$C_{p}$

$d$

E

I

$\langle I\rangle$

$k$

$l_{\text {tip }}$

$L$

$P$

$P_{\mathrm{o}}$

$P_{\mathrm{u}}$

$q$

$r$

$R$

$S_{\mathrm{d}}$

$S_{\mathrm{L}}$

$t$

$T_{\mathrm{b}}$

$T_{\mathrm{u}}$

$u_{\mathrm{b}}$

$u_{\mathrm{u}}$

$U$

$w_{\mathrm{f}}$

$x$

amplitude of curved flame

maximum amplitude of curved flame

flame surface area

speed of sound

specific heat under constant pressure

thickness of diffusion zone

activation energy

sound intensity or acoustic intensity

average sound intensity

wave number

flame tip position

tube length

acoustic pressure

acoustic pressure at bottom of tube

acoustic pressure in unburned mixture

heat flux

tube radius

gas constant

displacement flame velocity

laminar burning velocity

time

adiabatic flame temperature

temperature in unburned mixture

acoustic velocity in burned mixture

acoustic velocity in unburned mixture

flame velocity

maximum width of curved flame

distance from bottom of tube

\section{Greek Symbols}

$\alpha \quad$ thermal diffusivity

$\gamma \quad$ specific heat ratio

$\varepsilon \quad$ total energy per unit cross-sectional area

$\lambda$ wavelength of sound

$v \quad$ kinematic viscosity

$\xi_{\mathrm{u}} \quad$ displacement of unburned mixture

$\rho \quad$ density

$\rho_{\mathrm{b}} \quad$ density in burned mixture

$\rho_{\mathrm{u}} \quad$ density in unburned mixture

$\tau_{\mathrm{a}} \quad$ acoustic time

$1 / \tau_{\text {ins }} \quad$ growth rate of acoustic instability 


$\begin{array}{ll}1 / \tau_{\text {loss }} & \text { total acoustic loss } \\ 1 / \tau_{\text {rad }} & \text { radiative losses } \\ \tau_{\mathrm{t}} & \text { transit time } \\ 1 / \tau_{\text {wall }} & \text { wall losses } \\ \omega & \text { angular frequency }\end{array}$

\section{Dimensionless Numbers}

$\beta \quad$ dimensionless activation energy or Zel'dovich number

$K_{\text {re }} \quad$ reduced wave number

Le Lewis number

$M \quad$ Mach number $\left(=c / S_{\mathrm{L}}\right)$

$\operatorname{Pr} \quad$ Prandtl number $(=v / \alpha)$

$S \quad$ normalized flame surface area

$\operatorname{Tr} \quad$ transfer function defined by Ref. [11]

$U_{\mathrm{a}} \quad$ reduced acoustic velocity

\section{Subscript}

b

burned mixture

$\mathrm{f}$

flame

u

unburned mixture

o

bottom of tube 


\begin{abstract}
This paper deals with the onset mechanism of primary acoustic instability of downward-propagating flames in a combustion tube. We focus on the effects of a coupling constant, $\beta M$, where $\beta$ and $M$ represent the Zel'dovich and Mach numbers, respectively, and the variation in the flame surface area. To change the coupling constant, various gas compositions for lean ethylene flames diluted with carbon dioxide or nitrogen are used. We obtain a linear relationship between the coupling constant and the average acoustic intensity, and the critical values of the coupling constants are acquired through linear approximation regarding the onset of the primary acoustic instability. Furthermore, we adopt the $\mathrm{CO}_{2}$ laser irradiation method to alter the shape of the flame front, and experimental results show that the variation in the flame surface area does not always cause spontaneous generation of the primary acoustic instability in initially nonvibrating flames. Furthermore, even in initially vibrating flames, the growth rate of the primary acoustic instability is not associated with the growth or decline in the flame surface area in the present experiments. Finally, we also estimate the effects of acoustic losses on acoustic instability. Larger total acoustic losses tend to suppress acoustic vibration even at the same coupling constant. In addition, a remarkable transition from a secondary acoustic instability to the primary acoustic instability is observed at strong laser irradiation and is briefly discussed.
\end{abstract}

\title{
Keywords
}

Acoustic instability; Coupling mechanism; Growth rate; Lewis number; Reduced wave number 


\section{Introduction}

Interactions between combustion and acoustic vibration are of fundamental importance because industrial applications such as gas turbines and rocket engines often suffer when strong pressure disturbances develop. Research on such thermoacoustic instability has been conducted for a long time in various ways; experiments on a flame propagating in a tube have been the most elementarily adopted.

The experimental observations by Searby [1] constitute a typical example of research in a combustion tube. He reported four distinct regimes of downward-propagating flames in a tube: (1) a curved flame with no acoustic sound just after ignition; (2) a primary acoustic instability with a flat flame surface when the flame has reached the lower half of the tube; (3) a violent secondary acoustic instability with a corrugated flame; and (4) turbulent flame. The secondary acoustic instability has been distinctly studied experimentally $[2,3]$. The physical initiation mechanism of this instability is periodic acceleration of the flame separating dense and tenuous regions, which is similar to Faraday instability [4]. However, an important unsolved problem is the generation of the primary acoustic instability. An approach toward resolving this issue has generally been to consider the Rayleigh criterion [5]: an acoustic wave will be amplified if a time integral of the product of pressure and heat release fluctuations is positive over a pressure cycle. Considering this criterion, many previous researchers [6-12] have proposed various mechanisms on primary acoustic instability. We can classify them into two large groups depending whether they are related to coupling of the acoustic pressure and temperature fluctuations for a flat flame that is free from hydrodynamic instability or to fluctuation of a flame area caused by acoustic velocity fluctuation (heat generation coupled with variation in the flame surface area) for a curved flame arising from the well-known Darrieus-Landau (D-L) instability.

Initially, Dunlap [7] theoretically showed that the effect of the pressure and adiabatic temperature of an incoming acoustic wave modulates local heat release rates of a flame with the correct phase relationship. More recently, Clavin et al. [8] treated Dunlap’s mechanism in detail using a one-step high-activation energy flame. They reported that the growth rate of the primary acoustic instability of planar flames is proportional to the coupling constant $\beta M$, and the flames may overcome the damping mechanism due to heat transfer and viscous friction at the tube wall and to 
acoustic radiation losses at the open end of the tube when the coupling constant is adequately large $\left(\beta M \approx 10^{-2}\right.$ ). These explanations can be understood by the fact that for well-defined ranges of $\beta M$, the acoustic pressure more effectively contributes to the adiabatic temperature fluctuation since the Zel'dovich number is a nondimensional measure of the temperature sensitivity of the reaction rate. However, for sufficiently large Mach numbers, $M \geq 5 \times 10^{-3}$, a strong acoustic instability cannot appear because the transit time is much shorter than the acoustic time, $\tau_{\mathrm{t}} \ll \tau_{\mathrm{a}}$. Thus, the violent acoustic instability is predicted to appear at sufficiently small Mach numbers, $M \approx 10^{-3}$, for $\tau_{\mathrm{t}} / \tau_{\mathrm{a}}=$ $O(1)$ when $\beta$ is sufficiently large. Unfortunately, this mechanism subjected to the coupling with pressure and temperature has limitations, which can be applied only for planar, rather than curved, flames, and the initial growth rate of the acoustic instability measured by experiments for nonplanar flames [12] is two to three orders of magnitude higher than that expected by the theoretical prediction of Clavin et al. [8]. Thus, this analysis is very hard to apply to real combustion experiments since propagating premixed flames in an actual combustor do not take on a planar structure owing to exposure to hydrodynamic instabilities (e.g., D-L instability and gravity effects). Nevertheless, there is ample room to appraise the coupling constant, $\beta M$, for fully developed flat flames in the primary acoustic region.

Another mechanism was proposed by Markstein [10], who suggested that the primary acoustic instability is generated from variations of the flame surface area. An analytical analysis of this mechanism was further performed by Pelcé and Rochwerger [11] in connection with the experiments conducted by Searby [1]. They studied the coupling with variation in the flame surface area produced by the presence of an initial curved structure due to D-L instability and demonstrated that the growth rate of the primary acoustic instability is proportional to $(a k)^{2}$, where $a$ is an amplitude and $k$ is a wave number of the curved flame. For this study, they used a more complete model for flame dynamics that adopted stability limits of the D-L instability [9] and gravity effects into the problem in order to estimate a situation close to that of real propagating flames. The fundamental principle of this mechanism can be understood as follows: the rate of change in the acoustic energy in the tube is a function of the pressure and the velocity fluctuations across a discontinuity surface. First, the sufficiently large amplitude of the wrinkled flame caused by D-L instability causes deviation of the flow lines in front of the flame, and velocity gradients across the flame increase by the curvature 
effect. Then, the increasing velocity gradients induce change in the flame surface area, which is in phase with pressure fluctuation, and the primary acoustic sound is generated. Finally, the variation in the flame surface area caused by the acoustic velocity field forces the amplification of the primary acoustic sound and leads to the stabilization of D-L instability [1, 2, 11]. Their calculation indicates that the maximum growth rate takes place in the lower half of the tube, which is in good agreement with Searby's experimental observations [1]. However, it should be noted that the amplitude of a curved flame in their research is too small to apply to an actual flame, since this analysis assumes that the maximum value is $a k=1$. In addition, the flame amplitude by $\mathrm{D}-\mathrm{L}$ instability changes in a nonlinear trend rather than the neutrally stable D-L mode, which Pelcé and Rochwerger [11] considered to have constant amplitude in their calculation. Thus, it is required to consider actual D-L instability to confirm the mechanism based on the variation in the flame surface area. However, $a$ and $k$ are very hard to control experimentally; because the initial curved flame is intrinsically unstable, even the effect of $a$ and $k$ is significant in the instability mechanism.

To solve this problem, we utilize the novel $\mathrm{CO}_{2}$ laser-irradiation method to control the shape of the freely propagating flames. Tsuchimoto et al. [13] investigated the oscillating motion of an upward-propagating flame in a tube induced by the $\mathrm{CO}_{2}$ laser irradiation method, which forms a convex flame surface with the desired dimension toward the unburned mixture. The external laser irradiation preheats an unburned mixture locally in front of a reaction zone, and the flame propagation velocity increases locally. Here, the flame front acquires a strongly curved shape. Park et al. [14] have conducted experiments on downward-propagating flames with $\mathrm{CO}_{2}$ laser light and have observed a unique deformation of the flame surface (called an "ice-cream flame” in present work) from a flat flame surface caused by the primary acoustic instability when the flame front is exposed to $\mathrm{CO}_{2}$ laser light. Since then, Taniyama and Fujita [15] have examined the criteria of a transition from the primary to the secondary acoustic instability induced by a short-term $\mathrm{CO}_{2}$ laser-irradiation method. In their research $[14,15]$, however, the growth rate of primary acoustic instability depending on variations of flame surface areas was not discussed.

Therefore, one of the purposes of this paper is to provide experimental evidence regarding the dependence of change in the coupling constant $\beta M$, and the effects of variation in the flame area on the primary acoustic instability of downward-propagating flames in a tube. Another objective is to 
investigate the effects of acoustic losses on the primary acoustic instability. Generally, the acoustic losses depend on the geometries of combustion chambers and on the nature of the gaseous medium. Thus, we present experimental observations of damping effects in different gaseous media with different thermal diffusivities and kinematic viscosities in an identical tube. Finally, a new finding from a remarkable experimental observation of a transition from the secondary acoustic instability to the primary acoustic instability with strong $\mathrm{CO}_{2}$ laser irradiation is briefly discussed.

\section{Background}

\subsection{Coupling mechanisms of primary acoustic instability}

In this section, we review previously proposed mechanisms $[8,11]$ for the generation of primary acoustic instability for propagating flames in tubes. Here, two different coupling mechanisms characterizing the feedback mechanism of the acoustic instability on flame fronts are introduced.

For the coupling with pressure and temperature [8], the time derivative of the total energy per unit cross-sectional area, $\mathrm{d} \varepsilon / \mathrm{d} t$, is given as follows:

$$
\frac{\mathrm{d} \varepsilon}{\mathrm{d} t}=\delta P\left(\delta u_{\mathrm{b}}-\delta u_{\mathrm{u}}\right)
$$

Here $\delta P$ is the pressure fluctuation, $\delta u$ is the velocity fluctuation and the subscripts $u$ and b identify unburned and burned mixtures, respectively. For the pressure jump across the flame, it is of order $\rho U^{2}$, where $\rho$ is the density and $U$ is the flame velocity, and it is much smaller than the variations in the acoustic wave, $\rho c^{2}$, where $c$ is the speed of sound. Thus, we can suppose that pressures can be equal on both sides of the flame. In Eq. (1), the main challenge is to link $\left(\delta u_{\mathrm{b}}-\delta u_{\mathrm{u}}\right)$ to $\delta P$. The gas expansion $\left(\delta u_{\mathrm{b}}-\delta u_{\mathrm{u}}\right)$ is proportional to the fluctuation of the rate of heat release per unit cross-sectional area, $\delta q$. For simplicity of calculation, a one-step reaction with a large activation energy is assumed, corresponding to $\beta \gg 1$. The Zel'dovich number, $\beta$, represents the sensitivity of chemical reactions to the variation in the maximum flame temperature, and the inverse of $\beta$ implies an effective dimensionless width of the reaction zone [16]. Thus, the chemical reaction occurs in a thin region since a typical order of magnitude is $\beta \approx 10$. The Zel'dovich number can be calculated [17] as follows:

$$
\beta=E\left(T_{\mathrm{b}}-T_{\mathrm{u}}\right) /\left(R T_{\mathrm{b}}^{2}\right)
$$

Here $E$ is the activation energy, $T$ is temperature, and $R$ is the gas constant. Peters and Williams [18] 
define the activation energy as follows:

$$
\frac{E}{R}=-\frac{\mathrm{d} 2\left[\ln \left(\rho_{\mathrm{u}} S_{\mathrm{L}}\right)\right]}{\mathrm{d}\left(1 / T_{\mathrm{b}}\right)} .
$$

The laminar flame velocity, $S_{\mathrm{L}}$, and the heat flux, $q$, are very sensitive to temperature variations. A small relative variation in the flame temperature induces a modification to $S_{\mathrm{L}}$ and $q$ of order unity:

$$
\frac{\delta T_{\mathrm{b}}}{T_{\mathrm{b}}}=O\left(\frac{1}{\beta}\right) \Longrightarrow \frac{\delta S_{\mathrm{L}}}{S_{\mathrm{L}}}=O(1) \text { and } \frac{\delta q}{q}=O(1) .
$$

In the conservation equation of enthalpy, the relative variation in temperature and pressure in an acoustic wave are of the same order of magnitude:

$$
\frac{\delta T}{T} \approx \frac{\delta P}{P} \approx \frac{\rho c \delta u}{P}=M\left(\frac{\delta u}{S_{\mathrm{L}}}\right)
$$

According to (4), this temperature fluctuation induces a modification on the order $\beta M\left(\delta u / S_{\mathrm{L}}\right)$. For a relative gas expansion across a discontinuity surface, one may expect the same order of magnitude for the relative velocity jump across the flame to be as follows:

$$
\frac{\delta u_{\mathrm{b}}-\delta u_{\mathrm{u}}}{S_{\mathrm{L}}} \approx \beta M\left(\frac{\delta u}{S_{\mathrm{L}}}\right)
$$

The energy per unit cross-sectional area, $\varepsilon$, which is stored in the acoustic mode of the tube, is of order $\left(\tau_{\mathrm{a}} \delta u \delta P\right)$. Therefore, the characteristic order of magnitude of the growth rate of such an acoustic instability, $1 / \tau_{\text {ins }} \equiv \varepsilon^{-1} \partial \varepsilon / \partial t$, according to (1) and (6) would be the following:

$$
\frac{1}{\tau_{\text {ins }}} \approx \frac{\beta M}{\tau_{\mathrm{a}}} \text {. }
$$

Thus, the growth rate of the acoustic instability of planar flames is proportional to the coupling constant $\beta M$, according to (7).

In the case of coupling with a variation in the flame area, Pelcé and Rochwerger [11] introduced a new transfer function defined as follows:

$$
\operatorname{Tr}=\frac{\left(\delta u_{\mathrm{b}}-\delta u_{\mathrm{u}}\right)}{\delta u_{\mathrm{b}}}
$$

When we consider the mass conservation equation crossing the flame, the amplitude of the outgoing wave in the burned mixture is determined by the following relation:

$$
\delta u_{\mathrm{b}}-\delta u_{\mathrm{u}}=\left(\frac{\rho_{\mathrm{u}}}{\rho_{\mathrm{b}}}-1\right) S_{\mathrm{L}} \delta S
$$

where $S_{\mathrm{L}}$ is the laminar burning velocity and $\delta S$ is the perturbation of the normalized flame area. This factor is proportional to $(a k)^{2}$ because of $\delta S \sim(a k)^{2}[11]$. 
Both mechanisms can be understood intelligibly as follows. The rate of heat released per unit cross-sectional area of the tube by the curved flame is $q=\rho_{\mathrm{u}} S_{\mathrm{L}} C_{\mathrm{p}}\left(T_{\mathrm{b}}-T_{\mathrm{u}}\right) S$. It follows that the fluctuations of heat release are proportional to the perturbation of the normalized flame area, $\delta S$, which is proportional to $(a k)^{2}$ because of the relation $S=1+\frac{1}{4}(a k)^{2}$ for the unperturbed curved flame [11]. However, for the flat flame, $\delta S$ is almost constant and the fluctuations of heat release are only proportional to variation in the temperature across the flame. Therefore, the Zel'dovich number plays an important role in the acoustic instability of the flat flame.

\subsection{Acoustic losses}

In general, the acoustic energy is dissipated at the open end of the tube through radiative losses and at the wall through diffusive losses. The characteristic times of acoustic losses can be expressed as follows $[12,19]$.

Radiative losses: $1 / \tau_{\text {rad }}=(\omega r)^{2} /(8 c L)$, where $r$ is the tube radius and $L$ is the length of the tube. The radiative losses are proportional to $\omega^{2}$. In this study, the speed of sound, $c$, is adopted to be $267 \mathrm{~m} / \mathrm{s}$ in flames diluted with carbon dioxide and $349 \mathrm{~m} / \mathrm{s}$ in those diluted with nitrogen, which are the speeds of sound in pure $\mathrm{CO}_{2}$ and $\mathrm{N}_{2}$, respectively, at room temperature from the NIST database [20]. The radiative losses are only affected by the speed of sound when we use a single combustion tube. Therefore, it is expected that the flames diluted with nitrogen have higher $1 / \tau_{\text {rad }}$ than those diluted with carbon dioxide.

Wall losses: $1 / \tau_{\text {wall }}=\sqrt{8}(\omega \alpha)^{0.5}\left[(\gamma-1)+P r^{0.5}\right] / r$, where $\alpha$ is the thermal diffusivity, $\gamma$ is the specific heat ratio, $\operatorname{Pr}$ is the Prandtl number $(=v / \alpha)$, and $v$ is the kinematic viscosity. The first term in the brackets is related to the thermal boundary layer and the second term is related to the viscous layer. The wall losses are proportional to $(\omega \alpha+\omega v)^{0.5}$. Finally, the total acoustic loss, $1 / \tau_{\text {loss, }}$ is given by $1 / \tau_{\text {loss }}=1 / \tau_{\text {rad }}+1 / \tau_{\text {wall }}$.

\section{Experimental Procedure}

The experimental apparatus is schematically outlined in Fig. 1a. The propagation tube (transparent acrylic tube, 50-mm inner diameter and 511-mm length) is fixed vertically and charged with the tested gas at atmospheric pressure. To artificially change the flame area, we utilize the $\mathrm{CO}_{2}$ laser-irradiation method [13-15]. Ethylene gas plays an important role as the main absorption 
medium of $\mathrm{CO}_{2}$ laser light according to the NIST chemical database [20]. The $\mathrm{CO}_{2}$ laser beam (beam diameter: $3.3 \mathrm{~mm}$, SYNRAD Firestar v20, wave length: $10.6 \mu \mathrm{m}$ ) vertically passes along the center of the tube. A point ignition system using a spark plug is located near the upper end of the tube. When the spark igniter is activated, the top lid is simultaneously opened by an automatic opening system of the top lid of the tube powered by an electromagnet and a mechanical spring. The time-dependent behaviors of the downward-propagating flame are recorded by two high-speed cameras. One of the high-speed cameras (IDT MotionPro X-4 recording at $2000 \mathrm{fps}$ ) captures images from 230 to $290 \mathrm{~mm}$ from the upper side of the tube, as illustrated as the shaded region in Fig. 1b. In Searby's work [1], he observed that the primary acoustic sound occurs when the flame has reached the lower half of the tube. Therefore, half of the tube is an appropriate field to observe the generation process of the primary acoustic instability, and this captured region is called as "'view area” in the present work. Another high-speed camera (nac HSV-500C ${ }^{3}$ recording at $500 \mathrm{fps}$ ) captures whole images of the propagating flames in the tube. In cases of laser -irradiation, a flame front is exposed to laser light when the flames reach $230 \mathrm{~mm}$ from the upper end of the tube, and this moment is defined as $t=0.0 \mathrm{~ms}$ in the present study. Pressure temporal variation is measured with a PCB Piezotronics 106B52 dynamic pressure sensor located at the bottom end of the tube at a sampling rate of $10 \mathrm{kHz}$.

In this study, the premix gas composed of ethylene, oxygen, carbon dioxide, and nitrogen are set at atmospheric pressure in the tube. We test various gas compositions in association with different acoustic losses, laminar burning velocities, adiabatic flame temperatures, coupling constant $\beta M$, laser powers, and irradiation times shown in Table 1. The flame properties (e.g., laminar burning velocities and adiabatic flame temperatures) are calculated using CHEMKIN (Premix code/ USC II) [21].

To estimate the coupling mechanisms related to the coupling constant $\beta M$ for a planar flame leading to the generation of acoustic sound, we study the effects of some relevant parameters in various ways. Cases 1 and 2 are chosen to study the effect of damping factor on acoustic losses with the same laminar burning velocity. Comparison of Cases 1 and 3 as well as Cases 2 and 4 provide information on the effect of laminar burning velocity under the same damping ratios. The damping ratio has relevance to kinematic viscosity, thermal diffusivity, and acoustic frequency, as mentioned above. Cases 1, 5, and 6 allow estimation of the effect of adiabatic flame temperature with the same 
laminar burning velocity and damping ratio. Cases 1, 7, and 8 enable us to elucidate the effect of laminar burning velocity on primary acoustic instability with the same adiabatic flame temperature and damping ratio in more detail. In addition, in the comparisons between Cases 1, 9, 10, 11, and 12 as well as between Cases $4,13,14,15$, and 16, we can evaluate the influence of variations of the coupling constant $\beta M$ on the onset of primary acoustic instability under the same equivalence and damping ratios.

To take the coupling mechanisms featuring variations of flame areas for curved flames into account, we conduct experiments induced by $\mathrm{CO}_{2}$ laser irradiation. In this study, we only consider the deformed single cell structure to evaluate variations of flame surface areas qualitatively for a curved structure. Cases 2 and 4 are nonvibrating flames at initial conditions. Thus, they allow study of the potential for spontaneous generation of acoustic sound by increasing flame surface areas. Cases 1 and 4 provide experimental observations of the growth rate of the primary acoustic instability from initial vibrating flames (flat flames) to convex flames (ice-cream structure) under various laser powers. Finally, we can discuss the effects of growth or decline of the flame surface area on the growth rate of acoustic instability induced by a short-term $\mathrm{CO}_{2}$ laser-irradiation method in Case 17.

\section{Results and Discussion}

\subsection{Effects of direct coupling with pressure and temperature}

Figure 2 shows typical downward-propagating flat flame behavior after the initial curved flame in the primary acoustic instability in Case 1 . The numerical values at the bottom of each picture are the passage of time in the view area. A transition process from the initial curved flame to a flat flame is as follows. After ignition, a curved flame front propagates downward because of hydrodynamic instability. Then, the flame becomes flat under the presence of acoustic sound resonating at a quarter wave length. The transition from a curved flame to a flat flame occurs within $0.4 \mathrm{~s}$ after ignition. Figure 3 depicts temporal variation in the flame tip position, $l_{\text {tip }}$, and flame behavior (inserted pictures) with different gas compositions at the view area. Two distinct types of behavior are identified. First, a regime of acoustic instability in which the flames are flat and vibrating, as shown in Cases 1 and 4, is identified. Second, in a regime of nonacoustic instability, flames are curved and 
propagate without fluctuation, as shown in Cases 2 and 3. The cell size in Case 3 is relatively smaller than that in Case 2, and it is not homogeneous. Thus, it is apparent that measurement of the initial flame curvature is extremely difficult in experiments. On comparing Cases 1 and 2, the flame with relatively lower kinematic viscosity and thermal diffusivity (Case 1) displays periodic vibration, even with the same laminar burning velocity. Moreover, in Case 1, the mean flame velocity is lower than that in Case 2 because of the relatively small flame surface area. The mean flame velocities are 17.4 and $22.9 \mathrm{~cm} / \mathrm{s}$ in Cases 1 and 2, respectively. On comparing Cases 1 and 3, the flame with a higher burning velocity (Case 1) can only be observed as a vibration behavior even though both cases are at the same kinematic viscosity and thermal diffusivity. In the same way, the comparison of Cases 2 and 4 shows that a higher burning velocity tends to result in vibration behavior. As a summary of the above experimental observations, two facts can be pointed out: (1) larger kinematic viscosity and thermal diffusivity result in the suppression of acoustic vibration and (2) larger burning velocity tends to cause acoustic vibration.

The acoustic losses consist of the sum of the radiative losses and wall losses (see Section 2.2). In general, natural damping processes are unavoidable dissipations in flame propagation in one-end-open combustion tubes. For $1 / \tau_{\text {rad }}$, a faster speed of sound causes stronger acoustic radiation losses, which are considered in an identical combustion tube according to Section 2.2. For $1 / \tau_{\text {wall }}$, high thermal diffusivity leads to a gentle radial temperature gradient and an enhancement of the thermal-penetration depth at the flame edge, and the acoustic instability can be suppressed by acoustic energy dissipation with excessive heat loss to the acoustic boundary layer. Moreover, acoustic energy losses due to viscous friction should increase with kinematic viscosity. Therefore, it is probable that the flame cannot overcome natural damping with a high speed of sound, thermal diffusivity, and kinematic viscosity in Case 2. In another respect, the comparison of Cases 1 and 3 (Cases 2 and 4) shows the effect of the coupling constant $\beta M$ [8]; see also Section 2.1. Therefore, the temperature fluctuations associated with an incoming acoustic wave may be small (large) enough in comparison with the natural damping effect because the coupling constants are relatively small (large) in Case 3 (Case 4) under the same damping ratio.

To show the effect of a coupling constant on the growth rate of the primary acoustic instability more clearly, we conduct additional experiments that consider the effect of individual parameters 
included in the coupling constant $\beta M$, such as the adiabatic flame temperature and the laminar burning velocity. Fig. 4 illustrates the effects of the adiabatic flame temperature on (a) the flame tip position with time and (b) the average sound intensity with various adiabatic flame temperatures in Cases 1, 5, and 6. The inserted pictures show flame behaviors in different cases. The flames are relatively faster and have a broad amplitude with a higher adiabatic flame temperature in spite of the same laminar burning velocity as $22 \mathrm{~cm} / \mathrm{s}$ in Fig.4a. Moreover, the flame shape is closer to the flat surface as the adiabatic flame temperatures increase because of the relatively strong acoustic sound. For a quantitative estimation, we compare the coupling constant and the average sound intensity, $\langle I\rangle$.

The sound intensity (or the acoustic intensity) is defined as the acoustic power per unit area, $I=$ $P_{\mathrm{u}} u_{\mathrm{u}}$. The average sound intensity, $\langle I\rangle$, during time is given by [22]

$$
\langle I\rangle=\frac{1}{t} \int_{0}^{t} P_{\mathrm{u}}(t) u_{\mathrm{u}}(t) \mathrm{d} t
$$

Here, the acoustic pressure of the unburned mixture in front of the flame is obtained by the pressure measured at the bottom of tube, $P_{0}$. Pressures elsewhere in the tube at any time are given by [6]

$$
P_{\mathrm{u}}(x)=P_{\mathrm{o}} \cos \frac{2 \pi x}{\lambda}
$$

where $x$ is the distance along the tube measured from the bottom as depicted Fig. $1 \mathrm{~b}$ and $\lambda$ is the wavelength of sound associated with the angular frequency $\omega$. The displacement of the unburned mixture, $\xi_{\mathrm{u}}$, is given as follows [6]:

$$
\xi_{\mathrm{u}}(x)=\frac{P_{\mathrm{o}} \sin \frac{2 \pi x}{\lambda}}{\rho c \omega}
$$

Thus, we can obtain the acoustic velocity of the unburned mixture by $u_{\mathrm{u}}=\mathrm{d} \xi_{\mathrm{u}} / \mathrm{d} t$. In Fig. $4 \mathrm{~b}$, the average sound intensity, $\langle I\rangle$, given by Eq. (10) correlates well with the variations in the adiabatic flame temperatures.

Figure 5a illustrates the variation in the flame tip position with time in Cases 1, 7, and 8 . The flames have large amplitudes with higher laminar burning velocities, despite the same adiabatic flame temperature of 2,049 K. As shown in Fig. 4b, the average sound intensity increases with the laminar burning velocity in Fig. 5b. Moreover, the flame shapes are flat when the average sound intensities are larger than around $100 \mathrm{~W} \cdot \mathrm{m}^{-2}$, according to Figs. $4 \mathrm{~b}$ and $5 \mathrm{~b}$.

When experimental results in Figs. $4 \mathrm{~b}$ and $5 \mathrm{~b}$ are taken into consideration, the average acoustic intensity seems to be proportional to the adiabatic flame temperature and the laminar burning 
velocity. It is experimentally suggested that the coupling constant, $\beta M$, dominates the average sound intensity and a critical value of the coupling constant exists to attain a flat flame by acoustic vibration.

Additional experiments are conducted to clearly investigate the effect of the coupling constant. Figure 6 illustrates the temporal variation in the flame tip position for different coupling constants (a) diluted with $\mathrm{CO}_{2}$ and (b) diluted with $\mathrm{N}_{2}$ with the same equivalence ratios, respectively. In the case of $\mathrm{CO}_{2}$ dilution, the vibration motions are observed in three cases. Case 11 is the limit of acoustic sound detection, and then, flame vibrations are more clearly observed in Cases 12 and 1 as increasing coupling constant. In the case of $\mathrm{N}_{2}$ dilution, the vibration motion is observed in Cases 16 and 4, and Case 16 is the detection limit of acoustic vibration similar to Case 11. Both Figs. 6a and 6b show that increasing coupling constants tend to give increased amplitudes of vibrations.

We characterize the effect of the coupling constant on the primary acoustic instability according to the experimental observations shown so far. Figure 7 shows the relationship between $\beta M$ and average acoustic intensity, $\langle I\rangle$. The open symbols are the no-vibration cases and the solid symbols are the vibration cases. To determine the critical coupling constant for generating the primary acoustic instability, we establish a linear approximation between $\langle I\rangle$ and $\beta M$. The average acoustic intensity correlates well with the coupling constant. The best fit expressed by a solid line for lean ethylene diluted with $\mathrm{CO}_{2}$ is $\langle I\rangle=3.4 \times 10^{4}-301$ and the critical coupling constant, $\beta M_{\mathrm{CO} 2}$, is 0.0087. For lean ethylene diluted with $\mathrm{N}_{2}$, the best fit expressed by the dotted line is $\langle I\rangle=3.3 \times$ $10^{4}-322$ and the critical coupling constant, $\beta M_{\mathrm{N} 2}$, is 0.0098 .

A difference between $\beta M_{\mathrm{CO} 2}$ and $\beta M_{\mathrm{N} 2}$ may result from different damping ratios. In previous research [8], a more precise transfer function involves the damping rate. However, for simplicity of interpretation, we only adopt the coupling constant in this study, which may cause a difference in the critical coupling constants. It is noted that curved flames are included in the plots for acoustic vibration, while the analysis by Clavin et al. [8] was exclusively based on planar flames. Nevertheless, the amplitudes of pressure vibrations are fully developed even for curved flames in the measured area (view area), and the slopes of linear approximation are coherent even if comparison is made between curved and flat flames, as shown Fig. 7. Furthermore, the comparison between correlations for both $\mathrm{CO}_{2}$ dilution and $\mathrm{N}_{2}$ dilution suggests that the total acoustic losses tend to 
suppress acoustic intensity, even at the same coupling constant. In summary, the average acoustic intensity of the primary acoustic instability shows good correlation with the coupling constant, $\beta M$.

\subsection{Effects of coupling with variations of flame areas for curved flames}

To artificially change the flame surface area, a $\mathrm{CO}_{2}$ laser light is irradiated into the tube. The ethylene among the mixture components absorbs laser energy just in front of the flames. The local flame velocity should obviously increase due to the preheated unburned mixture by $\mathrm{CO}_{2}$ laser irradiation. Thus, the flame front is locally accelerated toward the unburned mixture (e.g., convex structure). The acceleration of the local flame velocity naturally increases as the applied laser power increases.

Figure 8 shows the temporal of the flame tip position for different gas compositions with various laser powers. Vertical dashed-dotted lines indicate beginning of laser irradiation. As we discussed in Section 4.1, Cases 1 and 4 were in the regime of acoustic instability, and Cases 2 and 3 were in the regime of nonacoustic instability without laser irradiation, as is apparent by the areas to the left of the dashed-dotted lines in Fig. 8. The sudden increase in the propagation speed starts around $50 \mathrm{~ms}$ after the beginning of laser irradiation. Similar to the cases without $\mathrm{CO}_{2}$ laser light, two distinct types of behavior are identified. First, the regime of acoustic instability in which the flames finally result in turbulent motions via ice-cream structures with vibrating motions in Cases 1 and 4; the typical flame behavior of transition processes in this regime will be discussed later. Second, the regime of nonacoustic instability in which flames have very large curved cells and propagate without fluctuation, even under continuous irradiation by $\mathrm{CO}_{2}$ laser light, as shown in Cases 2 and 3. Control of initial flame curvature by $\mathrm{CO}_{2}$ laser irradiation greatly facilitates qualitative evaluation on the effects of variation in the flame surface area, as shown in the inserted pictures (Cases 2 and 3) in Fig. 8. It also makes it possible to implement nonlinear growth of flame surface area, which may be given by hydrodynamic instability [23-25], as shown in Cases 2 and 3.

Pelce \& Rochwerger [11] gave the criterion for cellular thermoacoustic instability: the flame surface area should be sufficiently larger than the radius-to-length ratio of the combustion tube related to acoustic radiative losses:

$$
a k \geq r / L \text {. }
$$

However, the experimental results show that a sufficiently increased flame surface area due to laser 
irradiation does not support the generation of acoustic sound, as shown in Cases 2 and 3 in Fig. 8. The maximum values of $a k$ are 7.51 and $2.70(r / L \approx 0.05)$, and the maximum displacement flame velocities, $S_{\mathrm{d}}$, are 101.7 and $184.1 \mathrm{~cm} / \mathrm{s}$ in Cases 2 and 3 with laser powers of $18 \mathrm{~W}$, respectively. Here, $a$ and $k$ are calculated by $a=a_{\mathrm{f}}$ and $k=\pi / w_{\mathrm{f}}$, where $a_{\mathrm{f}}$ and $w_{\mathrm{f}}$ are the maximum amplitude and maximum width of curved flame, respectively, as shown in the inserted pictures in Fig. 8.

As shown in Fig. 8, Cases 1 and 4 are regimes of the primary acoustic instability even without laser irradiation. In these cases, the initial conditions are vibrating flat flames prior to the appearance of laser irradiation in the areas to the left of the dashed-dotted lines in Fig. 8. Now we consider Case 1. Figure 9a shows flame behaviors of transition from an initially flat flame to turbulent motion induced by a laser power of $18 \mathrm{~W}$ in Case 1 . One can divide the results into four individual types of flame behavior in order to describe the transition process in detail. First, the flat flame appears corresponding to the primary acoustic instability, as shown in Fig. 9a (I). The ice-cream flame is observed after exposure to $\mathrm{CO}_{2}$ laser light in Fig. 9a (II). In this region, we see the sudden acceleration of the flame tip position with increasing oscillation amplitude during 50-80 ms, as shown Fig. 8. After this, the flame exhibits a corrugated structure corresponding to the secondary acoustic instability in Fig. 9a (III). Once a concave structure appears at the flame tip, as shown in Fig. 9a (II-III), the corrugated wave propagates from the center to the outer edge of the flame as time goes by, as reported in the previous work $[14,15]$. Moreover, the slope of flame tip position suddenly decreases, as shown in Fig. 8. Finally, explosive turbulent motion appears with a breakdown of the curved structures in Fig. 9a (IV). In Case 1 with laser irradiation, the flames undergo the whole transition process as described above under every laser power.

Interestingly, in Case 4 with a laser power of $18 \mathrm{~W}$, we cannot observe turbulent motion because the corrugated structure (III) again becomes an ice-cream flame (II), although the flames are continuously exposed to $\mathrm{CO}_{2}$ laser light in Fig. 9b. This process (III-II) is repeated twice during the flame propagation. It is remarkable that the transition from secondary acoustic instability to primary acoustic instability takes place. This restabilization of the secondary acoustic instability may be caused by decreasing the reduced wave number due to the strong laser power. Here, the reduced wave number is defined by $K_{\mathrm{re}}=\pi d / w_{\mathrm{f}}$, where $d\left(=\alpha / S_{\mathrm{L}}\right)$ represents the thickness of the diffusion zone. This experimental result is consistent with the theoretical prediction [2]. According to the 
stability diagram for the secondary acoustic instability (the reduced acoustic velocity, $U_{\mathrm{a}}\left(=u_{\mathrm{u}} / S_{\mathrm{L}}\right)$, versus the reduced wave number) by Searby and Rochwerger [2], the cellular structures on the flame front due to hydrodynamic instability vibrates with small acoustic sound at low $U_{\mathrm{a}}$ and $K_{\mathrm{re}}$. When the reduced acoustic velocity increases, the hydrodynamic instability can be restabilized by acoustic acceleration $[1,2,11]$ and the cellular structures become flat with larger acoustic sound. When the reduced acoustic velocity further increases, the secondary acoustic instability spontaneously generates with the corrugated structure, followed by explosive acoustic sound and oscillation at half of the acoustic frequency for a sufficiently large reduced wave number. In our experiment, once the concave structure appears at the flame tip identical to Fig. 9a (II-III), the reduced wave number should directly increase and the secondary acoustic instability occurs [15]. However, the corrugated structure cannot be transferred to turbulent motion and returns the convex structure (ice-cream flame) in case of Fig. 9b (III-II). Because the corrugated flames are still exposed to $\mathrm{CO}_{2}$ laser light, the flame propagation enhanced by laser irradiation dominates the flame front structure resulting in one big cell, or very small wave number. Thereby, we can only observe the restabilization of the secondary acoustic instability at a laser power of $18 \mathrm{~W}$ in Case 4 (with turbulent motions appearing at laser powers of 6 and $12 \mathrm{~W}$, despite the same gas composition).

Meanwhile, another issue is that restabilization is not observed in the case of $\mathrm{CO}_{2}$ dilution. This can be understood as follows. According to the dispersion relation curve (the growth rate versus the wave number of perturbation) by Sivashinsky [24], the unstable region is expanded and the growth rate rapidly increases with decreasing Lewis number due to the curvature effect [23-25]. In the present work, the Lewis number in the case of $\mathrm{CO}_{2}$ dilution is relatively lower than in the case of $\mathrm{N}_{2}$ dilution $\left(L e_{\mathrm{CO} 2}=1.05\right.$ and $\left.L e_{\mathrm{N} 2}=1.35\right)$. Furthermore, the acoustic damping ratio in cases of $\mathrm{CO}_{2}$ dilution is smaller than $\mathrm{N}_{2}$ dilution. Therefore, the augmented flame propagation speed by $\mathrm{CO}_{2}$ laser light cannot restore the convex structure from the corrugation by the secondary acoustic instability in our experiments. Detailed analysis of restabilization of the secondary acoustic instability is beyond the scope of the present study and will be investigated in the future.

As a summary of the above experimental observations with laser irradiation, two facts can be pointed out: (1) the artificially deformed flame area does not always lead to spontaneous generation of the primary acoustic instability, and (2) the decreased reduced wave number due to strong laser 
irradiation could cause restabilization of the secondary acoustic instability.

To assess the effects of variations of flame areas on the growth rate of the acoustic instability, we have conducted estimation of pressure variation. Figure 10 shows the measured acoustic pressure fluctuation at the bottom of the tube as a function of time with a laser power of $18 \mathrm{~W}$ in Case 1 . Vertical dotted lines denote the boundaries of individual regions of transition processes from flat flames to turbulent motion. The start of laser irradiation is defined as $t=0.0 \mathrm{~s}$. No growth of pressure amplitude is observed until the corrugated structure appears, as shown Fig. 10. Once the corrugated structure via ice-cream flame appears as shown in Fig. 9a (III), the acoustic pressure exponentially increases. This is a typical feature of the secondary acoustic instability $[1,2,11]$; as more laser irradiation time elapses, turbulent motion appears with a breakdown of the coherent corrugated structures, and the acoustic pressure simultaneously decreases. An unexpected characteristic is the limited change in the pressure amplitude in the region of the ice-cream flame (II), even with a sudden change in the flame surface area. This phenomenon gives important information that the variation in the flame surface area is not always associated with pressure amplification. For further understanding, qualitative comparisons are given in Fig. 11. Figure 11a and b shows acoustic pressure in front of the flames and flame surface areas as a function of time before the corrugated structure appears (a) without laser irradiation and (b) with continuous laser irradiation at $18 \mathrm{~W}$ in Case 1 . The solid and dotted lines denote pressure change, $P_{\mathrm{u}}$, and flame surface area change, $A_{\mathrm{f}}$, respectively. Fluctuations of pressure and flame surface area are in phase at frequencies of 183 and $182 \mathrm{~Hz}$ in (a) and (b), respectively. The most important observation is that the acoustic pressure amplitudes are virtually the same, even though the flame surface area changes. Here, the maximum flame surface areas are 21.8 and $42.7 \mathrm{~cm}^{2}$ without and with laser irradiation in Case 1, respectively. In particular, for the case of laser irradiation, the maximum value of $(a k)^{2}$ is 12.3 .

To discuss the effects of flame surface area on pressure amplification, we also conduct an additional experiment on short-term laser irradiation. According to our previous research [15], the threshold of the secondary acoustic instability is given in terms of laser irradiation time and power. We select a case in which there is no transition to turbulent flame, namely the laser condition of 60 ms at $14 \mathrm{~W}$ for rich ethylene flame diluted with $\mathrm{CO}_{2}$ gas [15]. Figure 11c and d shows acoustic pressures in front of the flames and flame surface areas as functions of time (c) without laser 
irradiation and (d) with short-term laser irradiation in Case 17. In Fig. 11d, the start time of laser irradiation is defined as $t=0.0 \mathrm{~ms}$, and the vertical dashed-dotted line denotes the end of laser irradiation $(t=60 \mathrm{~ms})$. The deformation of the flame front starts at around $50 \mathrm{~ms}$ corresponding to an ice-cream flame. The peak flame area appears at around $140 \mathrm{~ms}$. In the images captured by a high-speed camera, the flame tip curvature starts to decrease at around $100 \mathrm{~ms}$, and finally, a flat flame is formed again. Fluctuations of pressures and flame surface areas are also in phase, and their frequencies are $168 \mathrm{~Hz}$ in (c) and (d). The acoustic pressure amplitudes are almost constant regardless of the growth or decline of flame surface areas. In the case of short-term laser irradiation, the acoustic pressure amplitude slightly changes with extreme variation in the flame surface area. Nevertheless, it can be ignored considering the order of magnitude of pressure amplification in the secondary acoustic instability, as shown in Fig. 10. Here, the maximum flame surface areas are 17.0 and $90.8 \mathrm{~cm}^{2}$ without and with a short-term laser irradiation in Case 17, respectively. In particular, for the case of laser irradiation, the maximum value of $(a k)^{2}$ is 9.9. In the experiment with a short-term laser irradiation, we obtain the same results as the cases of continuous laser irradiationthat is, the acoustic frequency and the growth rate of acoustic instability are not always associated with the increase or decrease in the flame surface area.

In this section, to confirm the mechanism of coupling with variation in the flame surface area first suggested by Markstein [10], we conduct various experiments with different flame surface areas induced by laser irradiation. The change in the flame area does not cause the spontaneous generation of the primary acoustic instability in initially nonvibrating flames. Furthermore, the variations of flame surface areas (growth or decline) do not support change to the acoustic frequency and growth rate of the acoustic pressure, even for initially vibrating flames.

\section{Conclusion}

This paper provides experimental evidence on the onset of the primary acoustic instability of downward-propagating flames in a tube. The main focus was on the coupling mechanism between pressure and temperature or coupling with variation in the flame surface area. The adiabatic flame temperature and the laminar burning velocity correlated well with the average acoustic intensity. Based on these results, we obtained linear relationships between the coupling constant and the 
average acoustic intensity, and the critical values of the coupling constants $\left(\beta M_{\mathrm{CO} 2}=0.0087, \beta M_{\mathrm{N} 2}=\right.$ 0.0098) were acquired through linear approximation of the onset of the primary acoustic instability for lean ethylene flames. Thus, the average acoustic intensity is proportional to the coupling constant, $\beta M$, on the primary acoustic instability in our experimental observations.

To ensure the relevance of the mechanism of coupling with variation in the flame area in the primary acoustic instability, we conduct additional experiments with $\mathrm{CO}_{2}$ laser irradiation. Contrary to the expectations based on previous research, the experimental results showed that artificial extreme growth of the flame area did not cause the spontaneous generation of the primary acoustic instability in initially nonvibrating flames. Even with initially vibrating flames, we could not observe the growth of acoustic pressure, even with a dramatic increase or decrease of the flame surface area by $\mathrm{CO}_{2}$ laser light irradiation. Therefore, we concluded that variation in the flame surface area does not always function as the coupling mechanism of the primary acoustic instability.

Finally, we also estimated the effects of acoustic losses on acoustic instability. Larger total acoustic losses as functions of speed of sound, kinematic viscosity, and thermal diffusivity tended to suppress the acoustic vibration even at the same coupling constant. In addition, a transition back from the secondary acoustic instability to the primary acoustic instability was found under strong laser irradiation. This remarkable restabilization of the secondary acoustic instability may be caused by the decreased reduced wave number as implied by the instability diagram given in the previous study [2].

\section{Acknowledgment}

This study was supported by a Grant-in-Aid for Scientific Research (KIBAN (B) \#26289042) from MEXT Japan. 


\section{Reference}

[1] G. Searby, Combust. Sci. and Tech. 81 (1992) 221-231.

[2] G. Searby, D. Rochwerger, J. Fluid Mech. 231 (1991) 529-543.

[3] G. H. Markstein, Nonsteady Flame Propagation, Pergamon (1964).

[4] M. Faraday, Phil. Trans. R. Soc. Lond. 121 (1831) 319-340.

[5] J. Rayleigh, Nature. 18 (1878) 319.

[6] W. E. Kaskan, Proc. Combust. Inst. 4 (1953) 575-591.

[7] R. A. Dunlap, Aeronautical Research Center, University of Michigan. Project MX833. Rep. UMM-43(1950).

[8] P. Clavin, P. Pelcé, L. He, J. Fluid Mech. 216 (1990) 299-322.

[9] P. Pelcé, P. Clavin, J. Fluid Mech. 124 (1982) 219-237.

[10] G. H. Markstein, Proc. Sixth Natl Congr. Appl. Mech., Cambridge, Mass. 11 (1970) 11-33.

[11] P. Pelcé, D. Rochwerger, J. Fluid Mech. 239 (1992) 293-307.

[12] C. Clanet, G. Searby, P. J. Clavin, Fluid Mech. 385 (1999) 157-197.

[13] M. Tsuchimoto, O. Fujita, T. Honko, Y. Nakamura, H. Ito, Proc. Combust. Inst. 32 (2009) 1003-1009.

[14] J. S. Park, O. Fujita, Y. Nakamura, H. Ito, Proc. Combust. Inst. 33 (2011) 1105-1112.

[15] Y. Taniyama, O. Fujita, Combust. Flame 161 (2014) 1558-1565.

[16] D. Bradley, Internal Combustion Engineering: Science and Technology (J Wearing, Ed.), Elsevier Applied Science, London (1990) 287-331.

[17] P. Clavin, Prog. Energy Combust. Sci. 11 (1985) 1-59.

[18] N. Peters, F. A. Williams, Combust. Flame 68 (1987) 185-207.

[19] L. Landau, E. Lifshitz, Fluid Mechanics, 1st edn. Pergamon (1986).

[20] The national institute of standards and technology (NIST), available at $<$ hittp://webbook.nist.gov/chemistry/>.

[21] R. J. Kee, R. M. Rupley, et al., CHEMKIN PRO, Reaction Design, Inc., San Diego, CA, 2009.

[22] S. Gade, M. Sc, Sound intensity, Technical review 3 (1982) 3-39.

[23] S. H. Kang, S. W. Baek, H. G. Im, Combust. Theor. Model. 10 (2006) 659-681.

[24] G. I. Sivashinsky, Rev. Fluid. Mech. 15 (1983) 179-199.

[25] J. Yuan, Y. Ju, C. K. Law, Combust. Flame 144 (2006) 386-397. 


\begin{tabular}{|c|c|c|c|c|c|c|c|c|c|c|c|c|c|}
\hline Case & $\mathrm{C}_{2} \mathrm{H}_{4}$ & $\mathrm{O}_{2}$ & $\mathrm{CO}_{2}$ & $\mathrm{~N}_{2}$ & $\phi$ & $\begin{array}{c}S_{\mathrm{L}} \\
(\mathrm{cm} / \mathrm{s}) \\
\end{array}$ & $\begin{array}{c}T_{\mathrm{b}} \\
(\mathrm{K}) \\
\end{array}$ & $\begin{array}{c}\alpha \\
\left(\mathrm{cm}^{2} / \mathrm{s}\right) \\
\end{array}$ & $\begin{array}{c}v \\
\left(\mathrm{~cm}^{2} / \mathrm{s}\right) \\
\end{array}$ & $\begin{array}{c}1 / \tau_{\text {loss }} \\
\left(\mathrm{s}^{-1}\right) \\
\end{array}$ & $\beta M$ & $\begin{array}{c}\text { w/ or w/o } \\
\text { laser }\end{array}$ & $\begin{array}{c}\text { vibration } \\
\text { or not } \\
\end{array}$ \\
\hline 1 & 0.071 & 0.24 & 0.69 & & 0.90 & 22 & 2049 & 0.13 & 0.10 & 8.2 & 0.012 & \multirow{4}{*}{$\begin{array}{c}\text { with laser } \\
(0,6,12 \\
18(\mathrm{~W}))\end{array}$} & $\mathrm{O}$ \\
\hline 2 & 0.040 & 0.14 & & 0.82 & 0.84 & 22 & 1832 & 0.21 & 0.15 & 12.7 & 0.007 & & $x$ \\
\hline 3 & 0.059 & 0.20 & 0.74 & & 0.90 & 11 & 1814 & 0.13 & 0.09 & 8.1 & 0.005 & & $x$ \\
\hline 4 & 0.049 & 0.18 & & 0.77 & 0.84 & 40 & 2092 & 0.21 & 0.15 & 12.7 & 0.013 & & $\mathrm{O}$ \\
\hline 5 & 0.067 & 0.25 & 0.68 & & 0.80 & 22 & 1988 & 0.13 & 0.10 & 8.2 & 0.010 & \multirow{12}{*}{$\begin{array}{l}\text { without } \\
\text { laser }\end{array}$} & $\mathrm{O}$ \\
\hline 6 & 0.063 & 0.27 & 0.67 & & 0.70 & 22 & 1927 & 0.13 & 0.10 & 8.3 & 0.009 & & $\mathrm{O}$ \\
\hline 7 & 0.070 & 0.26 & 0.67 & & 0.80 & 25 & 2049 & 0.13 & 0.10 & 8.2 & 0.012 & & $\mathrm{O}$ \\
\hline 8 & 0.069 & 0.29 & 0.64 & & 0.70 & 29 & 2049 & 0.14 & 0.10 & 8.3 & 0.014 & & $\mathrm{O}$ \\
\hline 9 & 0.061 & 0.20 & 0.74 & & 0.90 & 12 & 1851 & 0.13 & 0.09 & 8.1 & 0.006 & & $x$ \\
\hline 10 & 0.063 & 0.21 & 0.73 & & 0.90 & 14 & 1900 & 0.13 & 0.09 & 8.1 & 0.007 & & $x$ \\
\hline 11 & 0.066 & 0.22 & 0.71 & & 0.90 & 16 & 1951 & 0.13 & 0.09 & 8.2 & 0.008 & & $\mathrm{O}$ \\
\hline 12 & 0.068 & 0.23 & 0.70 & & 0.90 & 19 & 2000 & 0.13 & 0.09 & 8.2 & 0.010 & & $\mathrm{O}$ \\
\hline 13 & 0.041 & 0.15 & & 0.81 & 0.84 & 22 & 1850 & 0.21 & 0.15 & 12.7 & 0.007 & & $x$ \\
\hline 14 & 0.043 & 0.15 & & 0.81 & 0.84 & 26 & 1900 & 0.21 & 0.15 & 12.7 & 0.008 & & $x$ \\
\hline 15 & 0.044 & 0.16 & & 0.80 & 0.84 & 29 & 1950 & 0.21 & 0.15 & 12.7 & 0.009 & & $x$ \\
\hline 16 & 0.046 & 0.16 & & 0.79 & 0.84 & 32 & 2000 & 0.21 & 0.15 & 12.7 & 0.010 & & $\mathrm{O}$ \\
\hline 17 & 0.090 & 0.20 & 0.71 & & 1.35 & 12 & 1817 & 0.13 & 0.09 & 8.1 & 0.004 & $\begin{array}{c}\text { with laser } \\
\text { during } 60 \mathrm{~ms} \\
(0,14(\mathrm{~W}))\end{array}$ & $\mathrm{O}$ \\
\hline
\end{tabular}

Table. 1 Summary of results for the tested gas 


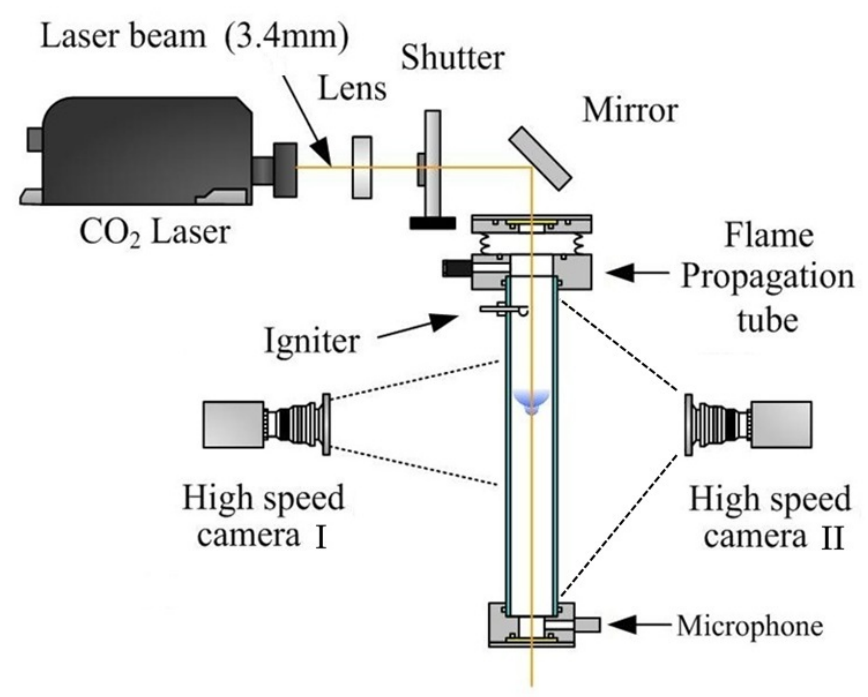

(a)

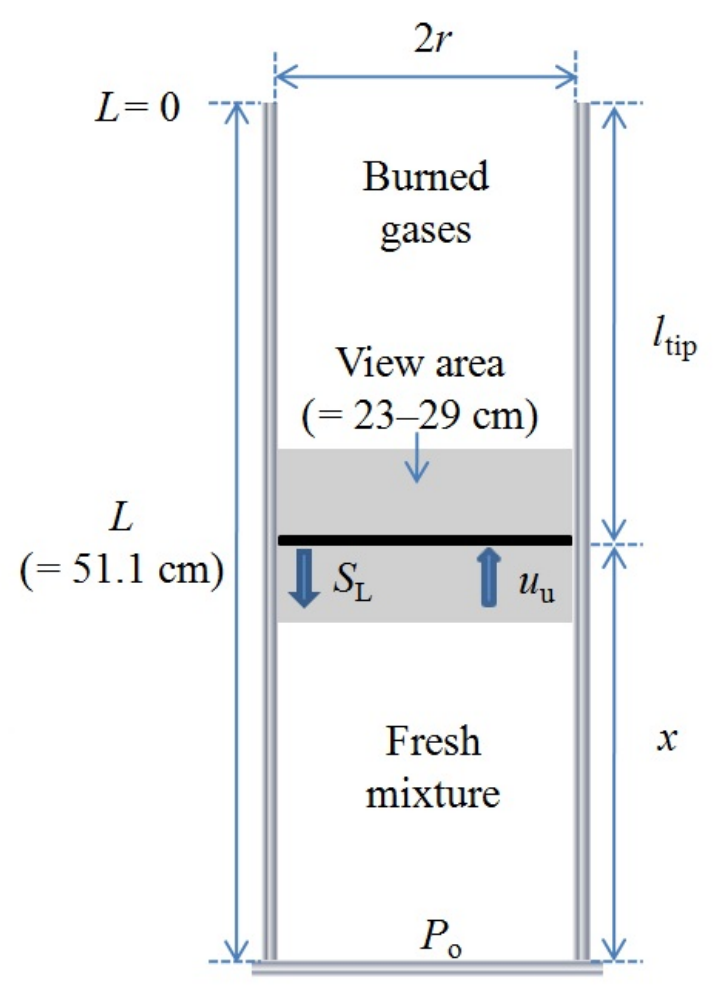

(b)

Fig. 1. (a) Schematic drawing of the experimental setup and (b) general configuration 


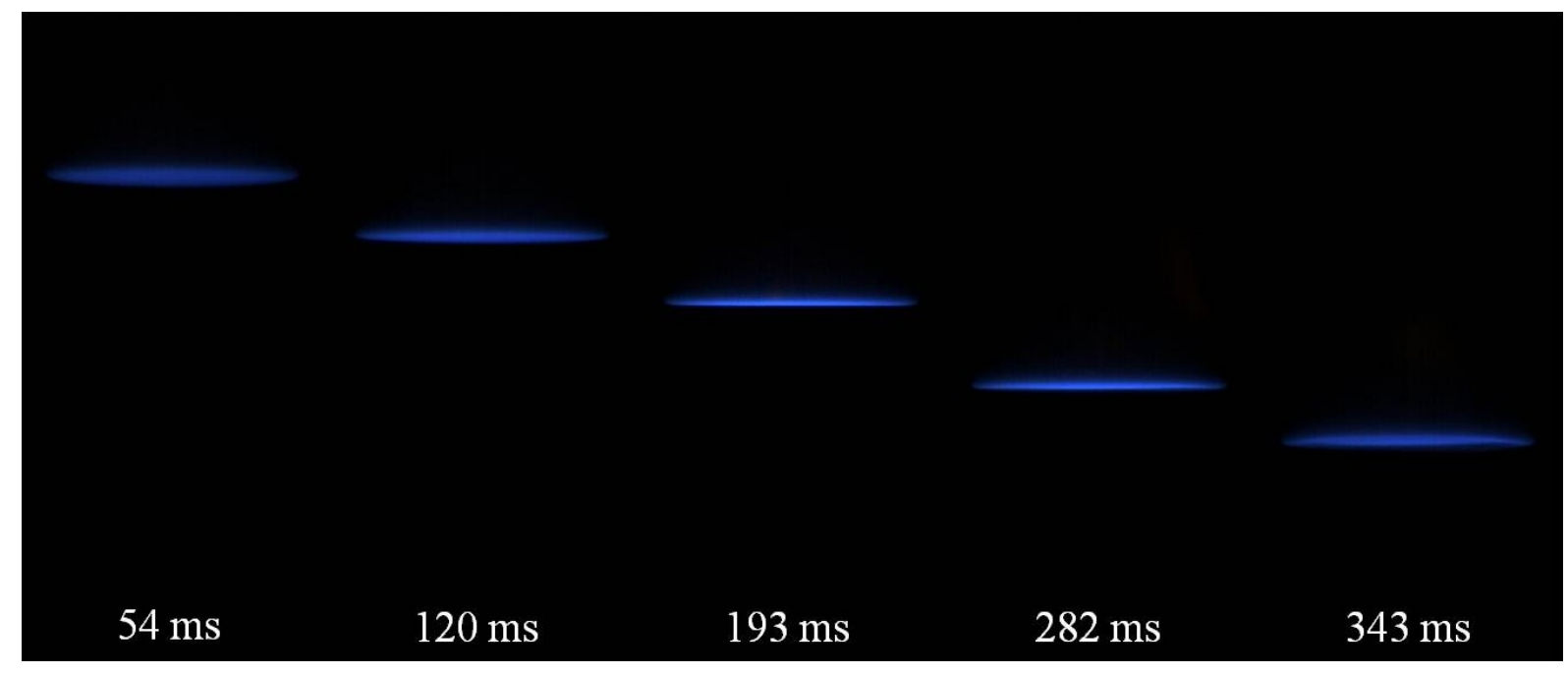

Fig. 2. Sequential images for a propagating flame without laser power in Case 1 


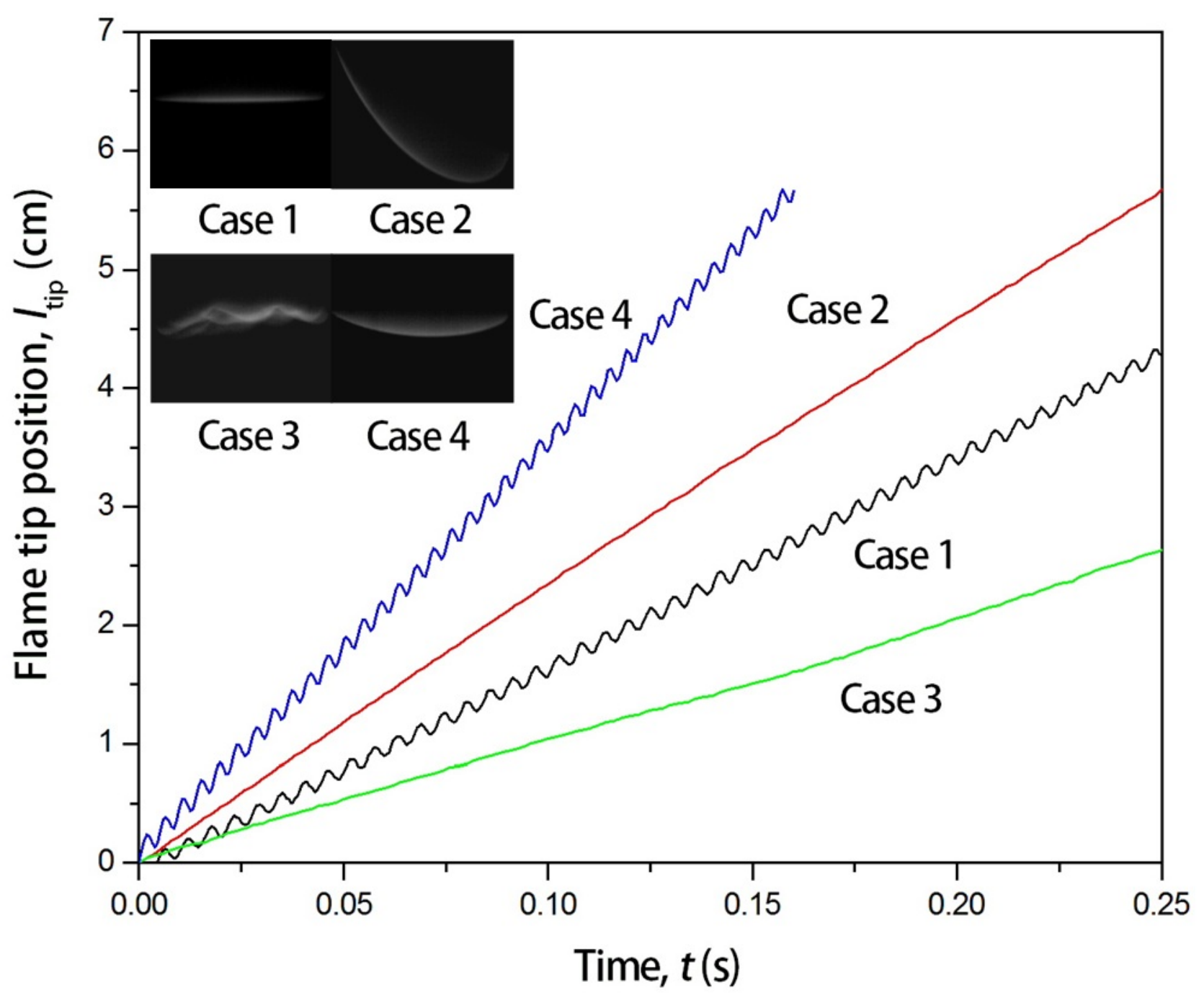

Fig. 3. Temporal flame tip position without laser irradiation in Cases 1, 2, 3, and 4 


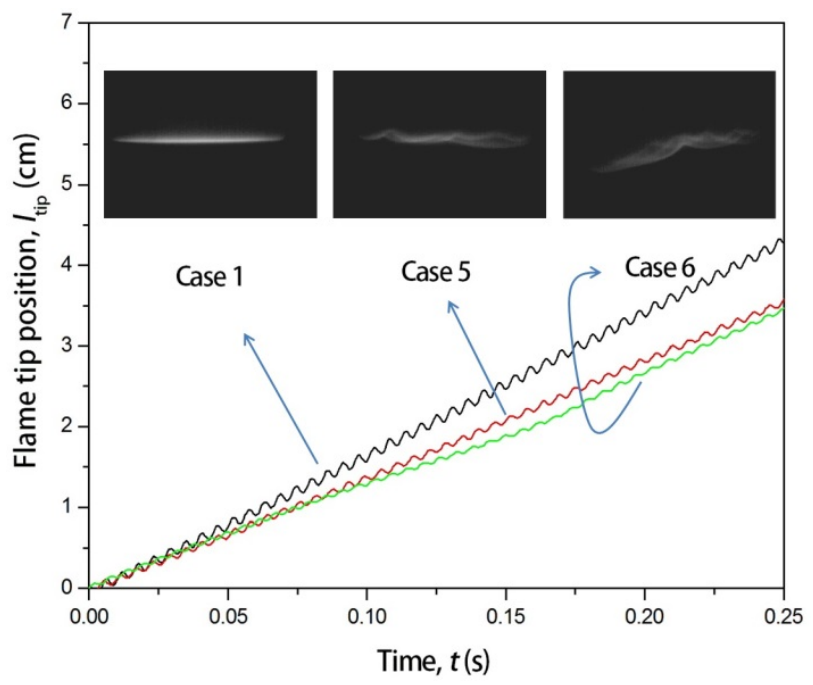

(a)

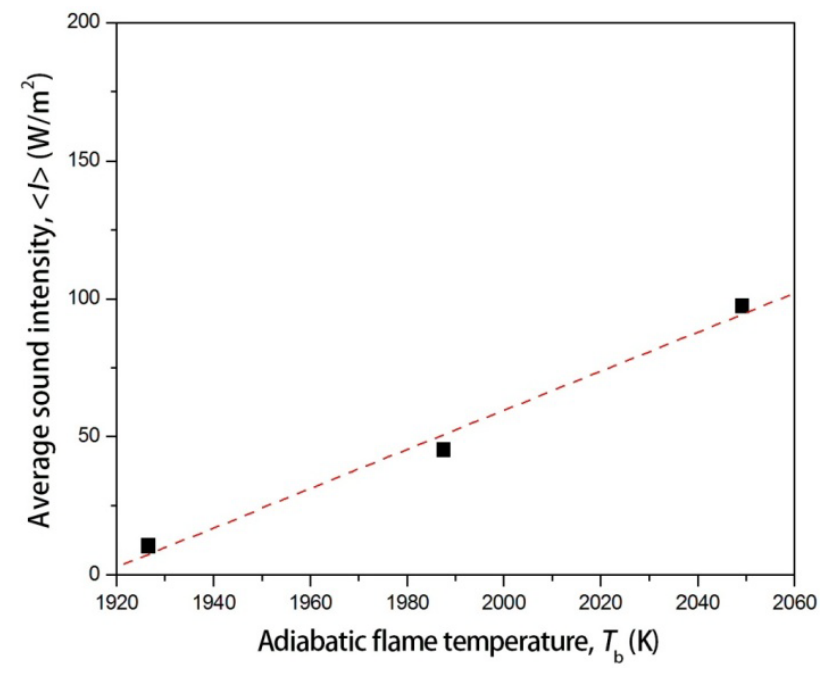

(b)

Fig. 4. Effect of adiabatic flame temperature on primary acoustic instability (a) temporal flame tip position without laser irradiation and (b) average sound intensity with various adiabatic flame temperatures in Cases 1, 5, and 6. 


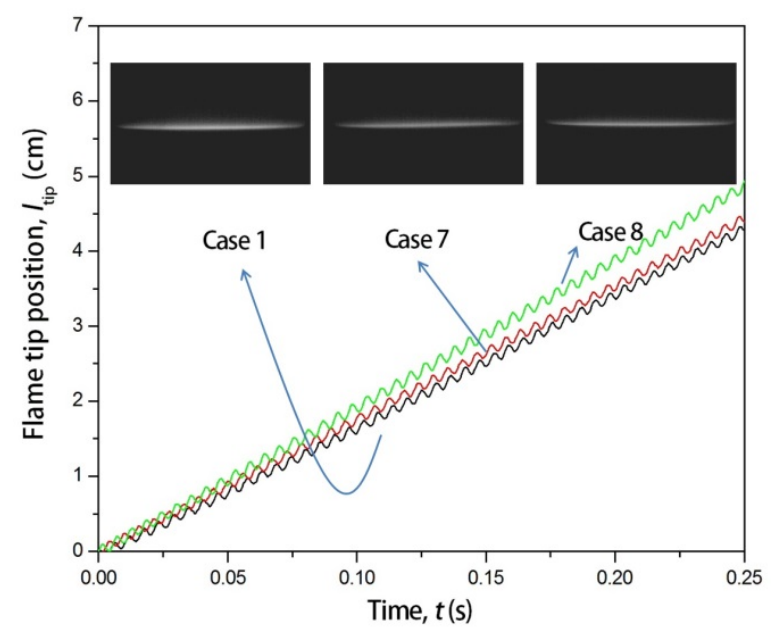

(a)

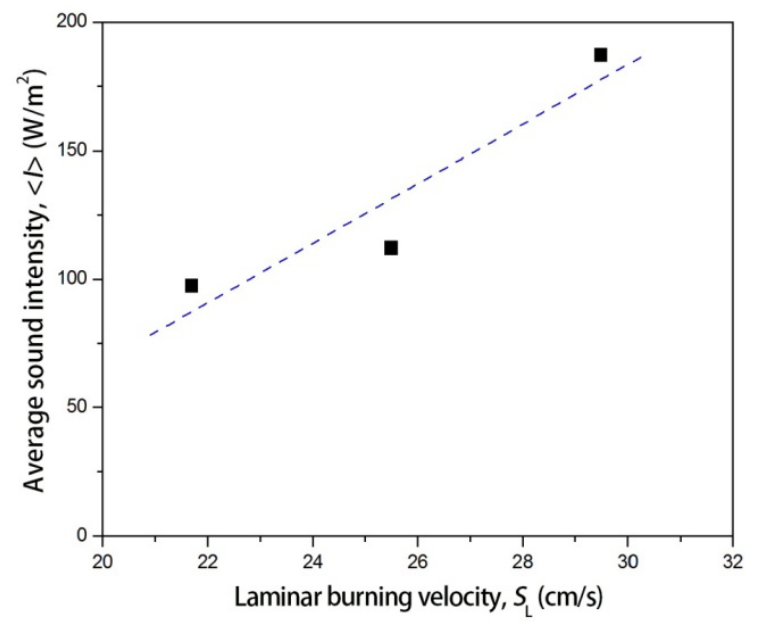

(b)

Fig. 5. Effect of laminar burning velocity on primary acoustic instability

(a) temporal flame tip position without laser irradiation and (b) average sound intensity with various laminar burning velocities in Cases 1, 7, and 8. 


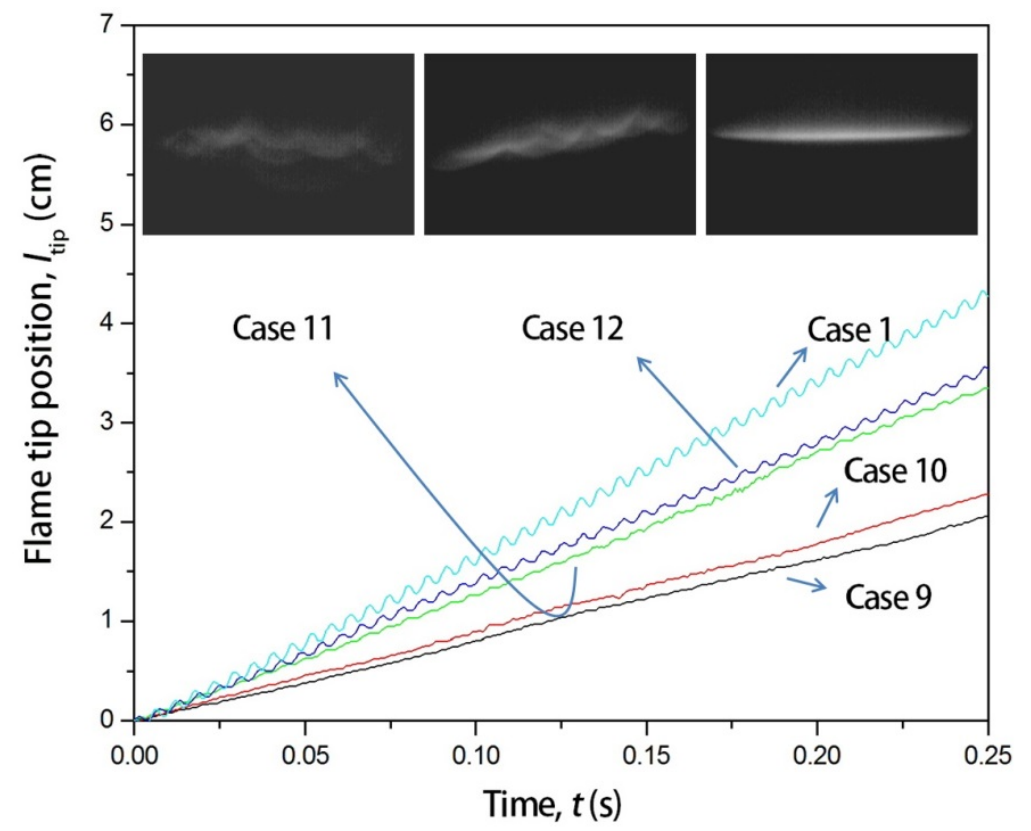

(a)

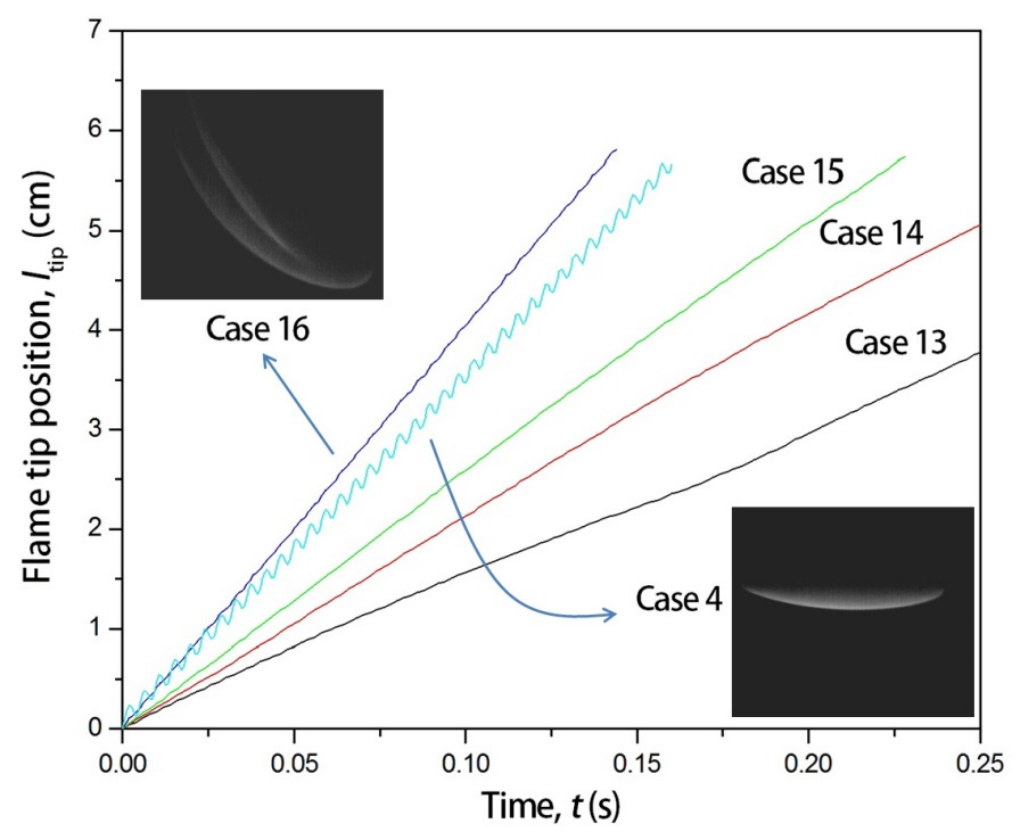

(b)

Fig. 6. Temporal flame tip positions without laser

(a) diluted with $\mathrm{CO}_{2}$ at $\phi=0.90$; (b) diluted with $\mathrm{N}_{2}$ at $\phi=0.84$. 


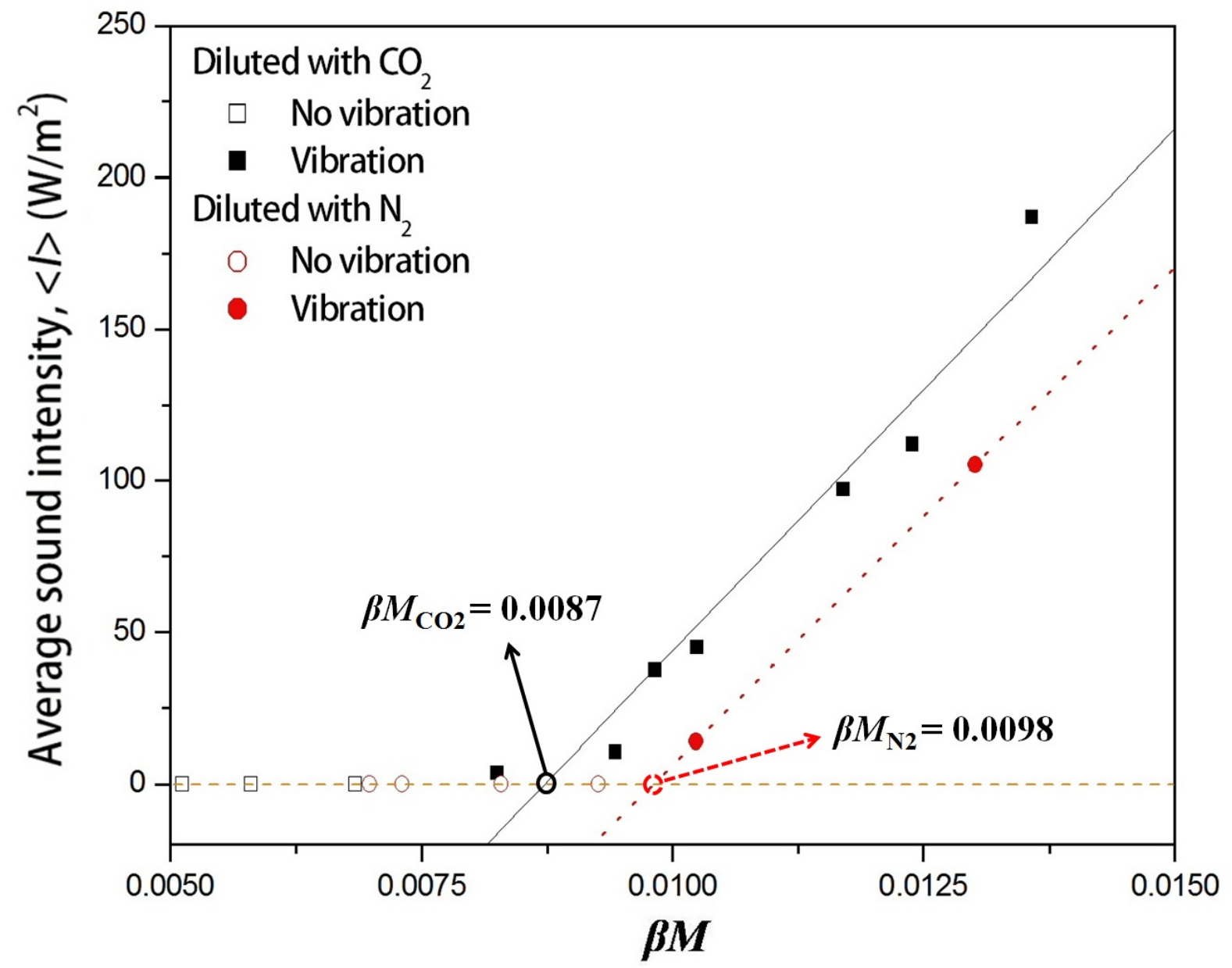

Fig. 7. Characteristics of the onset of the primary acoustic instability

Dependence of the average sound intensity for primary acoustic instability on the coupling constant at various gas compositions. 


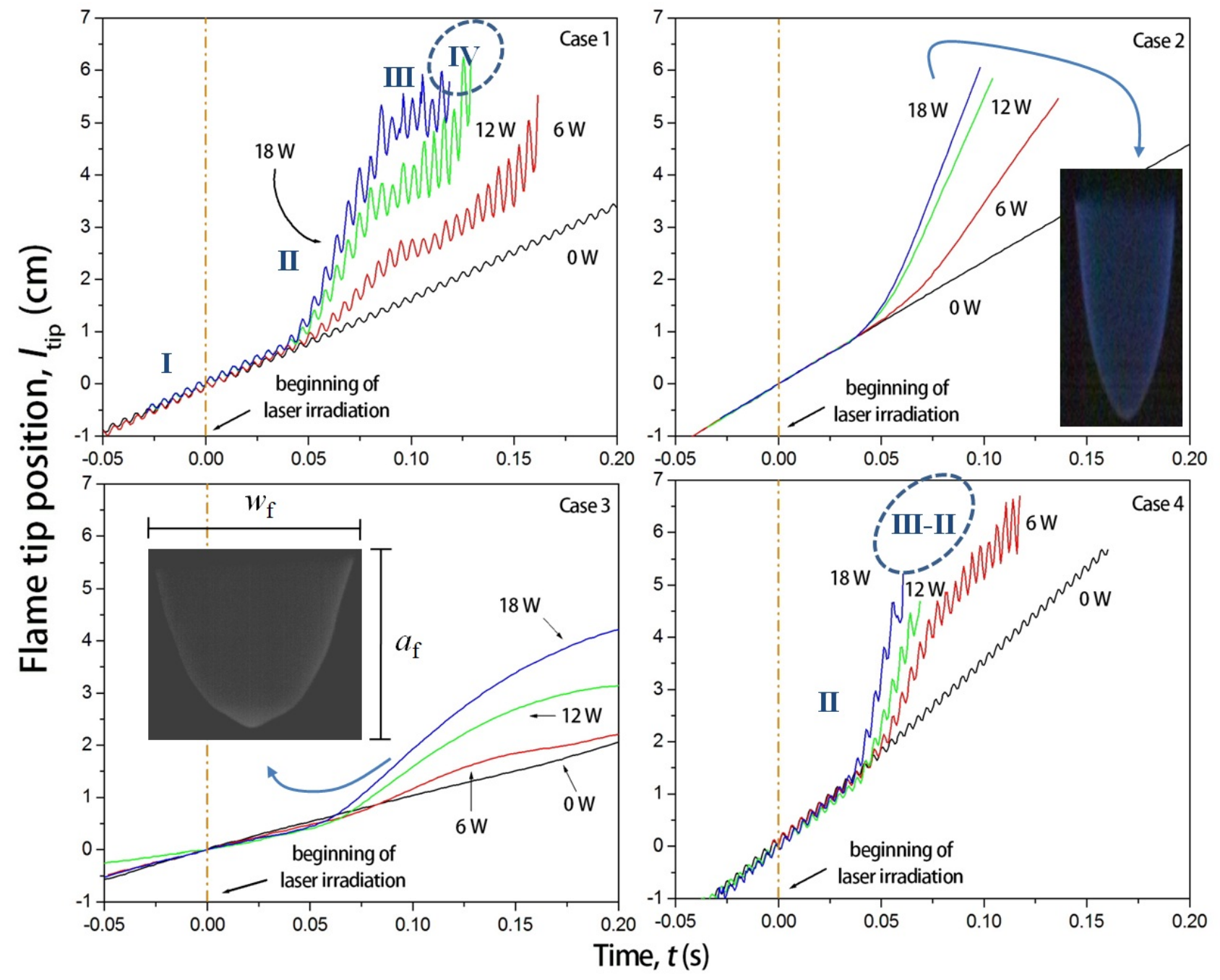

Fig. 8. Temporal flame tip position with various laser power inputs with flame propagating downward in Cases 1, 2, 3, and 4 


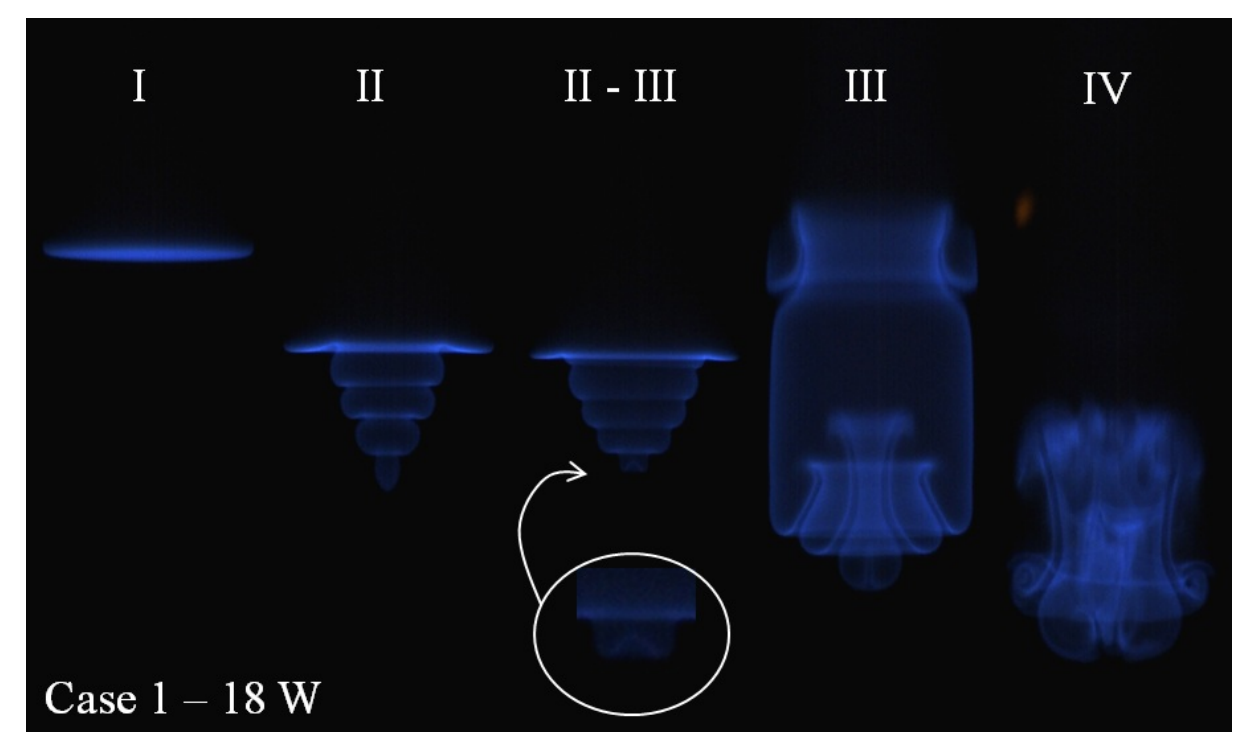

(a)

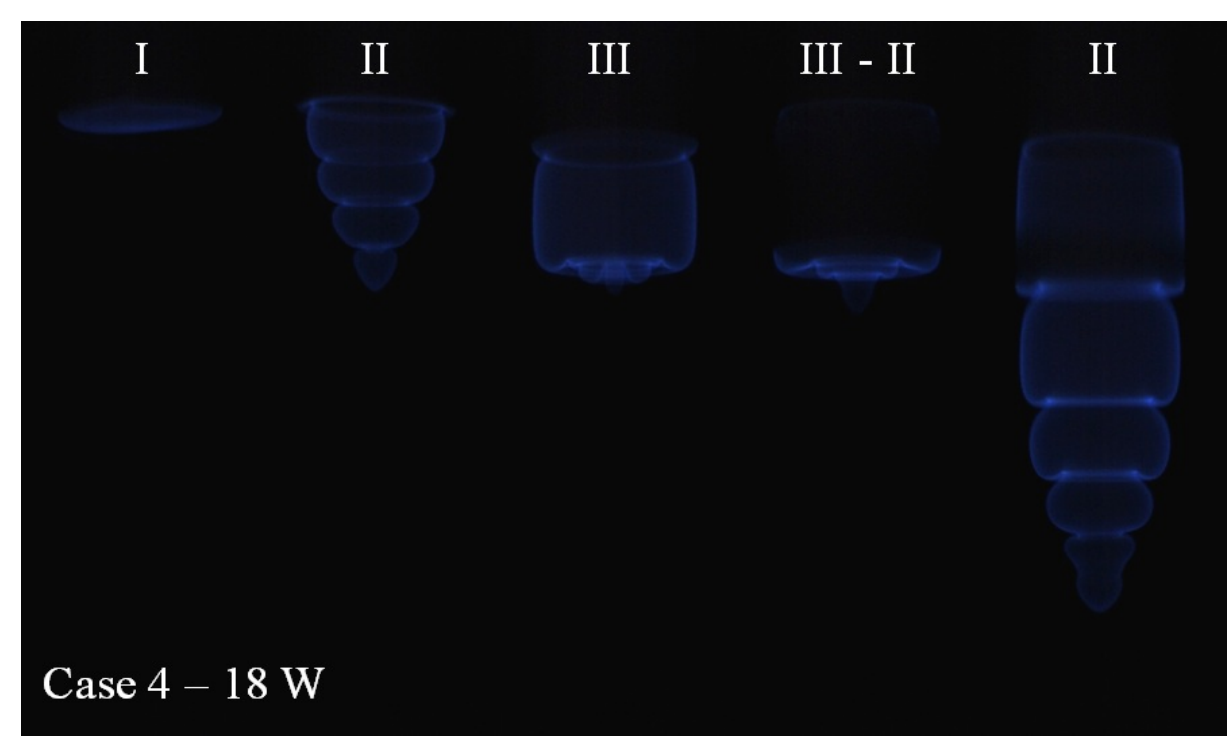

(b)

Fig. 9. Two distinctive flame behaviors of transition with laser power of $18 \mathrm{~W}$ in (a) Case 1 and (b) Case 4

(I) flat flame, (II) ice-cream flame, (III) corrugated structure, and (IV) turbulent motion. 


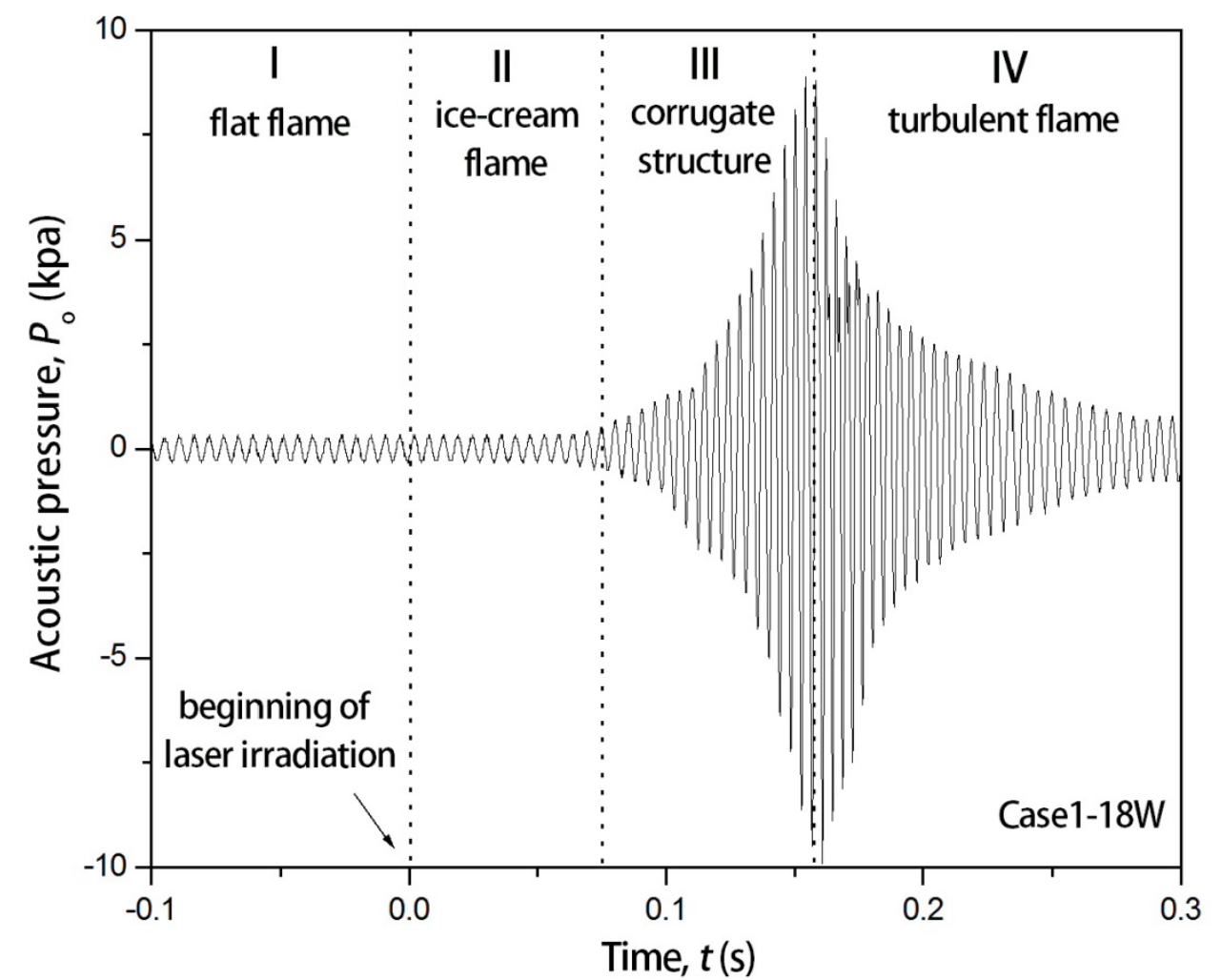

Fig. 10. Acoustic pressure at the bottom of the tube as a function of time with a laser power of $18 \mathrm{~W}$ in Case 1 


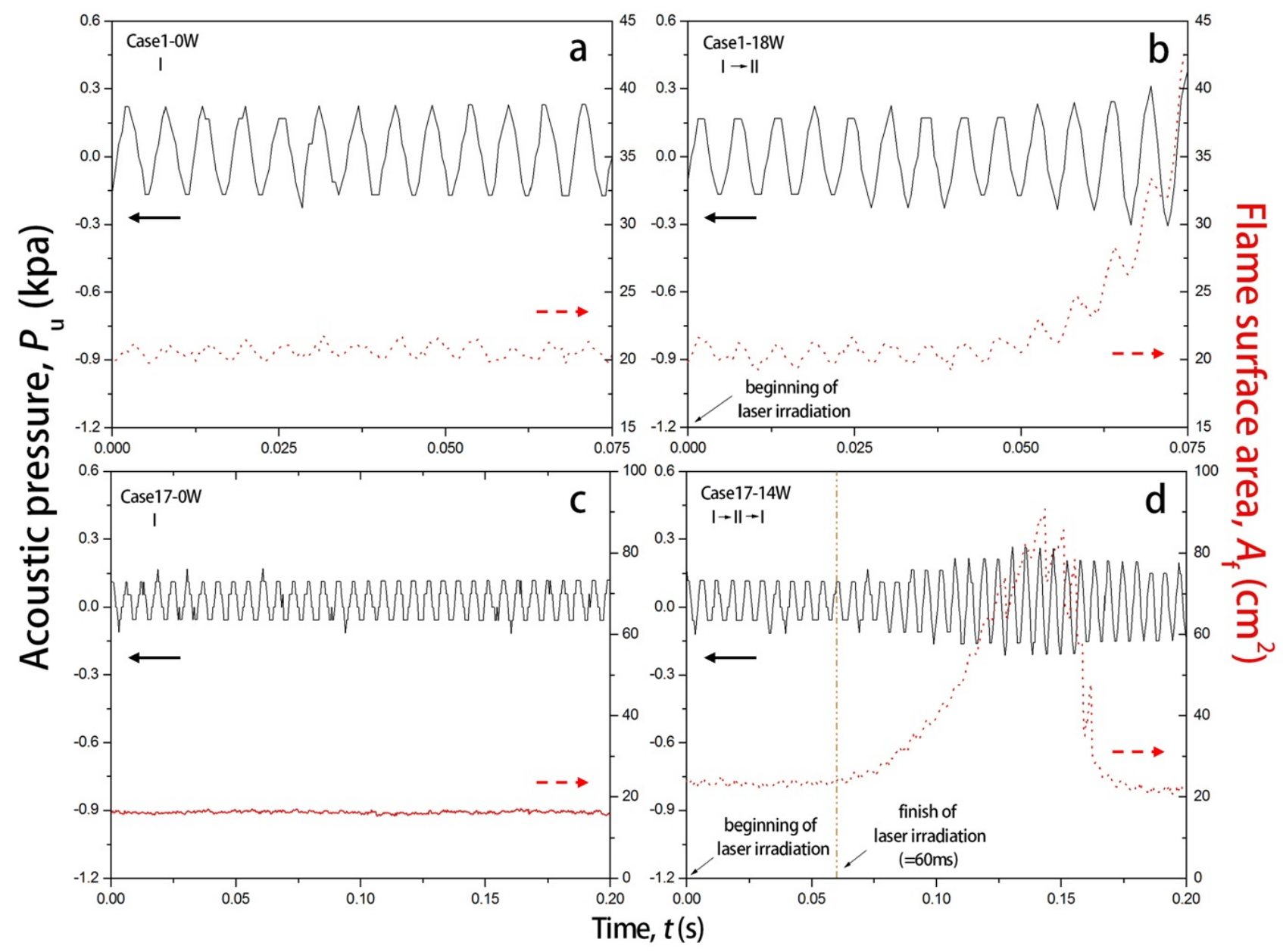

Fig. 11. Acoustic pressure in front of the flames and flame surface area as functions of time (a) without laser irradiation and (b) with continuous laser irradiation at $18 \mathrm{~W}$ in Case 1; and (c) without laser irradiation and (d) with a short-term laser irradiation over $60 \mathrm{~ms}$ at $14 \mathrm{~W}$ in Case 17 